\title{
Предоперационная визуализация
}

и интраоперационная навигация новообразований околощитовидных желез при первичном гиперпаратиреозе

\author{
К.Ю. Слащук ${ }^{1}$ М.В. Дегтярев ${ }^{1}$, П.О. Румянцев ${ }^{2}$, А.К. Еремкина ${ }^{1}$, Н.В. Тарбаева ${ }^{1}$, Д.Г. Бельцевич ${ }^{1}$, \\ И.В. Ким ${ }^{1}$, Г.А. Мельниченко ${ }^{1}$, Н.Г. Мокрышева ${ }^{1}$ \\ 'ФГБУ «Национальный медицинский исследовательский центр эндокринологии» Минздрава России; Россия, 117063 Москва, \\ ул. Дмитрия Ульянова, 11; \\ ${ }_{2}^{2}$ Межхуународный медицинский центр «СОГАЗ МЕДИЦИНА»; Россия, 191186 Санкт-Петербург, ул. Малая Конюшенная, 8
}

К он та к ты : Константин Юрьевич Слащук slashuk911@gmail.com

Введение. Первичный гиперпаратиреоз представляет собой одно из самых распространенных эндокринных заболеваний. По частоте встречаемости он стоит после сахарного диабета и патологий щитовидной железы. Ранняя диагностика и лечение первичного гиперпаратиреоза позволяют избежать тяжелых поражений костей скелета, почек, других органов и систем и тем самым уменьшить частоту инвалидизации, улучшить качество жизни пациентов. Единственным радикальным методом лечения данного заболевания является хирургическое удаление патологически измененных, гиперфункционирующих околощитовидных желез.

Цель исследования - повысить эффективность предоперационной топической диагностики и интраоперационной навигации образований околощитовидных желез.

Материалы и методы. В исследование включены 200 пациентов с лабораторно подтвержденным гиперпаратиреозом, проходивших предоперационную топическую диагностику (ультразвуковое исследование, планарную сцинтиграфию и однофотонную эмиссионную компьютерную томографию, совмещенную с компьютерной томографией (ОФЭКТ/КТ), в ряде случаев дополненных КТ с контрастным усилением или тонкоигольной аспирационной биопсией со смывом из иглы на паратгормон) и получивших хирургическое лечение в период с 2017 по 2020 г. Проведено одномоментное открытое сравнительное исследование, в ходе которого оценивались клинические, лабораторные и инструментальные данные пациентов. Период наблюдения после хирургического лечения по поводу первичного гиперпаратиреоза составлял не менее 6 мес.

Результаты. Хирургическое лечение в объеме минимально инвазивной паратиреоидэктомии выполнено 189 больным из 200 включенных в исследование пациентов. При использовании комбинации методов визуализации (ультразвукового исследования околощитовидных желез и ОФЭКТ/КТ, дополненного КТ с контрастным усилением и/или тонкоигольной аспирационной биопсией со смывом из иглы на паратгормон) общая точность выявления образований околощитовидных желез составила 99 \% (95 \% доверительный интервал (ДИ): 97-100), диагностическая специфичность - 77 \% (95 \% ДИ: 54-100), диагностическая чувствительность - $100 \%$ (95\% ДИ: 98-100), прогностическая ценность положительного и отрицательного результатов - 98 \% (95 \% ДИ: 97-100) и 100 \% (95 \% ДИ: 98-100) соответственно. Использование одноканального гамма-зонда с ${ }^{99 \mathrm{~m} T с-М И Б И ~ п р и ~ м и н и м а л ь н о ~ и н в а з и в н о и ̆ ~ п а р а т и р е о и д-~}$ эктомии позволяет интраоперационно судить о радикальности хирургического лечения по снижению уровня излучения в операционном поле и сохранению его в удаленном образовании околощитовидной железы.

Заключение. Результаты нашего исследования позволили разработать алгоритм предоперационной топической диагностики образований околощитовидных желез у пациентов с лабораторно верифицированным первичным гиперпаратиреозом и показаниями к хирургическому лечению. На 1-м этапе рекомендуется выполнять ультразвуковое исследование щитовидной железы и околощитовидных желез, на 2-м этапе при отсутствии выраженных изменений в щитовидной железе - планарную сцинтиграфию и ОФЭКТ/КТ с $99 \mathrm{mT}$ функциональной или структурной патологии щитовидной железы - КТ с внутривенным контрастированием, которую при необходимости можно дополнить тонкоигольной аспирационной биопсией со смывом из иглы на паратгормон.

Ключевые слова: первичный гиперпаратиреоз, визуализация околощитовидных желез, медицинская визуализация, ультразвуковое исследование, планарная сцинтиграфия, однофотонная эмиссионная компьютерная томография, совмещенная с компьютерной томографией 
Для цитирования: Слащук К.Ю., Дегтярев М.В., Румянцев П.О. и др. Предоперационная визуализация и интраоперационная навигация новообразований околощитовидных желез при первичном гиперпаратиреозе. Опухоли головы и шеи 2021;11(4):10-21. DOI: 10.17650/2222-1468-2021-11-4-10-21.

\title{
Preoperative imaging and intraoperative navigation of the parathyroid glands neoplasms in primary hyperparathyroidism
}

\author{
K. Yu. Slashchuk, M.V. Degtyarev ${ }^{1}$, P.O. Rumyantsev ${ }^{2}$, A.K. Eremkina ${ }^{1}$, N.V. Tarbaeva ${ }^{1}$, D.G. Beltsevich ${ }^{1}$, I.V. Kim ${ }^{1}$, \\ G.A. Melnicthhenko ${ }^{1}$, N.G. Mokrysheva ${ }^{1}$ \\ ${ }^{1}$ Nationl Medicl Research Centre of Endocrinology, Ministry of Health of Russia; 11 Dmitriya Ulyanova St., Moscow 117036, Russia; \\ ${ }^{2}$ International Medical Center "SOGAZ”; 8 Malaya Konyushennaya St., St. Petersburg 191186, Russia
}

Contacts: $\quad$ Konstantin Yuryevich Slashchuk slashuk911@gmail.com

Introduction. Primary hyperparathyroidism is one of the most common diseases of the endocrine system, after diabetes mellitus and thyroid pathologies. Early diagnosis and treatment of primary hyperparathyroidism allow avoiding severe damage to the bones, kidneys, other organs, thereby reducing the incidence of disability and improving the patients quality of life. The only radical treatment for primary hyperparathyroidism is the surgical removal of the pathologically altered, hyperfunctioning parathyroid glands.

The study objective - to increase the efficiency of preoperative topical diagnosis and intraoperative navigation of parathyroid glands.

Materials and methods. 200 patients with laboratory-verified primary hyperparathyroidism, who underwent preoperative topical diagnostics (ultrasound, planar scintigraphy and single-photon emission computed tomography, combined with computed tomography (SPECT/CT), in some cases supplemented with contrast enhanced CT with/or fine needle aspiration biopsy with flushing from a needle on for parathyroid hormone) and received surgical treatment, in period from 2017 to 2020. A single-stage, open-label comparative study was carried out, including clinical, laboratory and instrumental data of patients. The follow-up period after surgery for primary hyperparathyroidism was at least 6 months. Results. From 200 included patients, surgical treatment in the amount of minimally invasive parathyroidectomy was performed in 189 patients. As a result of the analysis of the diagnostic accuracy, for a combination of ultrasound and SPECT/CT with augmented contrast enhanced CT, the overall accuracy in visualizing of parathyroid glands was $99 \%(95 \%$ confidence interval (CI): 97-100), diagnostic specificity $77 \%$ (95\% CI: 54-100), sensitivity $100 \%$ (95\% CI: 98-100), the predictive value of positive and negative results was $98 \%$ (95\% CI: 97-100) and $100 \%$ (95\% CI: 98-100) respectively.

Conclusion. The results allowed us to develop an algorithm for preoperative topical diagnosis of parathyroid glands in patients with laboratory-verified primary hyperparathyroidism and indications for surgical treatmen. We recommend to perform ultrasound of the thyroid and parathyroid glands in all patients at the first stage, in the absence of evident changes in the thyroid gland, at the second stage - scintigraphy and SPECT/CT with ${ }^{99 \mathrm{~m} T C-M I B I}$; in cases with significant concomitant functional or structural pathology of the thyroid gland - contrast enhanced CT; if necessary, supplementing fine needle aspiration biopsy with flushing from a needle on for parathyroid hormone.

Key words: Primary hyperparathyroidism, visualization of parathyroid glands, medical imaging, ultrasound, sestamibi scintigraphy, single-photon emission computed tomography, combined with computed tomography

For citation: Slashchuk K.Yu., Degtyarev M.V., Rumyantsev P.O. et al. Preoperative imaging and intraoperative navigation of the parathyroid glands neoplasms in primary hyperparathyroidism. Opukholi golovy $i$ shei $=$ Head and Neck Tumors 2021;11(4):10-21. (In Russ.). D0I: 10.17650/2222-1468-2021-11-4-10-21.

\section{Введение}

По данным Российского регистра по первичному гиперпаратиреозу (ПГПТ), заболеваемость ПГПТ в Российской Федерации на 2017 г. составила в зависимости от региона 1,3-7,6 случая на 100000 взрослого населения в год [1]. Показатели распространенности ПГПТ значительно варьируют по всему миру, что обусловлено сложностями в диагностике его ранних форм, наличием нормокальциемического варианта данного заболевания и недостаточной осведомленностью врачей о нем. В среднем ПГПТ выявляется у 0,5-1 \% людей трудоспособного возраста и у $2 \%$ представителей стар- шей возрастной группы (55-75 лет). Чаще это заболевание встречается у женщин [2, 3]. Более чем в 85-90\% случаев оно является спорадическим и обусловлено солитарной аденомой околощитовидной железы (ОЩЖ). В $10-15 \%$ случаев встречаются множественные аденомы и/или гиперплазия нескольких ОЩЖ, примерно в $1 \%$ случаев наблюдается рак ОЩЖ. Основную сложность представляют образования атипично расположенных ОЩЖ, которые составляют до 15-20 \% всех случаев ПГПТ, а также множественное поражение ОЩЖ, в том числе как один из компонентов синдрома множественных эндокринных неоплазий (МЭН-синдрома). 
Поскольку хирургическое лечение является единственным радикальным методом лечения ПГПТ, точная дооперационная топическая диагностика образований ОЩЖ на сегодняшний день остается актуальной проблемой. Показатели информативности и диагностической точности всех известных методов визуализации, описанных в литературе, значительно отличаются в различных медицинских учреждениях и воспроизводимы среди центров экспертного уровня [4-6]. Выбор одного оптимального исследования крайне затруднителен, поскольку ни одно из них не обладает $100 \%$-ной диагностической точностью. К тому же каждый метод имеет ограничения и, безусловно, всегда в значительной степени операторозависим [7, 8]. Важными факторами, обусловливающими выбор последовательности и метода топической диагностики образований ОШЖ, являются оснащенность клиники и опыт специалистовдиагностов. Хотя использование нескольких методов визуализации материально затратно для пациента, оно позволяет с большей долей вероятности предоперационно определить локализацию измененных ОЩЖ. В совокупности с использованием интраоперационной навигации это дает возможность максимально повысить эффективность и безопасность хирургического лечения (с перспективой долгосрочной нормализации кальция в крови, уменьшения частоты рецидивов и хирургических осложнений) [9-11].

Таким образом, более широкое внедрение комбинированных методов визуализации позволит повысить эффективность и безопасность хирургического лечения ПГПТ по сравнению с ревизией областей типичного расположения ОЩЖ и уменьшить частоту хирургических осложнений и рецидивов/персистенции ПГПТ.

Цель исследования - повысить эффективность предоперационной топической диагностики и интраоперационной навигации образований ОЩЖ.

\section{Материалы и методы}

Дизайн исследования. Работа выполнена в дизайне одномоментного сравнительного исследования. Выборка формировалась сплошным способом. Ее размер предварительно не рассчитывался. План обследования пациентов включал сбор анамнеза жизни, заболевания и семейного анамнеза, физикальное обследование, лабораторную и инструментальную диагностику. На следующем этапе во всех случаях (если это было возможно) проводилось хирургическое лечение в объеме минимально инвазивной паратиреоидэктомии с последующим гистологическим исследованием послеоперационного материала и динамическим наблюдением за пациентами в течение не менее 6 мес. для исключения рецидива/персистенции ПГПТ (рис. 1).

Оценивалась диагностическая информативность (диагностическая чувствительность (ДЧ), диагностическая специфичность (ДС), общая точность (ОТ), прогностическая ценность положительного результата (ПЦПР) и прогностическая ценность отрицательного результата (ПЦОР)) методов топической визуализации: ультразвукового исследования (УЗИ), планарной сцинтиграфии, однофотонной эмиссионной компьютерной томографии, совмещенной с компьютерной томографией (ОФЭКТ/КТ), КТ или ОФЭКТ/КТ с контрастным усилением, тонкоигольной аспирационной биопсией (ТАБ) со смывом из иглы на паратгормон (ПТГ), изолированно друг от друга и в комбинации. Интервал между различными диагностическими исследованиями во всех случаях составлял не более 2 мес.

Критерии соответствия. В исследование были включены пациенты с лабораторно подтвержденным ПГПТ и показаниями к хирургическому лечению в соответствии со стандартными алгоритмами [12]: ПТГ $\geq 65$ пг/мл в сочетании с повышенным уровнем общего или альбумин-скорректированного кальция в крови (превышение нормы на 0,25 ммоль/л и более) и с гипофосфатемией или нормальным уровнем фосфора в крови.

Критерии исключения из исследования:

- тяжелые сопутствующие заболевания (декомпенсация хронической сердечной недостаточности, хроническая обструктивная болезнь легких, печеночная/почечная недостаточность и др.);

- прием препаратов, потенциально влияющих на фосфорно-кальциевый обмен (препараты лития, бисфосфонаты и др.);

- вторичный ГПТ (ВГПТ);

- отказ от участия в исследовании на любом этапе.

Условия проведения. Все пациенты, включенные в исследование, проходили обследование и хирургическое лечение в ФГБУ «НМИЦ эндокринологии» Минздрава России в период с 2017 по 2020 г. В качестве кандидатов на участие в исследовании рассматривались больные старше 18 лет, обратившиеся в Центр или направленные в него из других его подразделений с диагнозом «ПГПТ».

Описание медицинского вмешательства. Лабораторные исследования были выполнены в клинико-диагностической лаборатории ФГБУ «НМИЦ эндокринологии» Минздрава России. Для подтверждения диагноза ПГПТ у всех пациентов определяли уровень интактного ПТГ в сыворотке крови с использованием иммунохимического анализатора Cobas 6000 (Roche, Швейцария), а также уровни кальция общего, ионизированного и креатинина с использованием биохимического анализатора Architect c8000 (Abbott, США). При обнаружении повышения содержания кальция в крови его уровень измеряли повторно.

При планировании исследования в рутинный перечень не был включен анализ уровня альбумина крови, необходимого для расчета альбумин-скорректированного кальция. Вместо этого определяли уровень ионизированного кальция. Количество кальция в суточной 


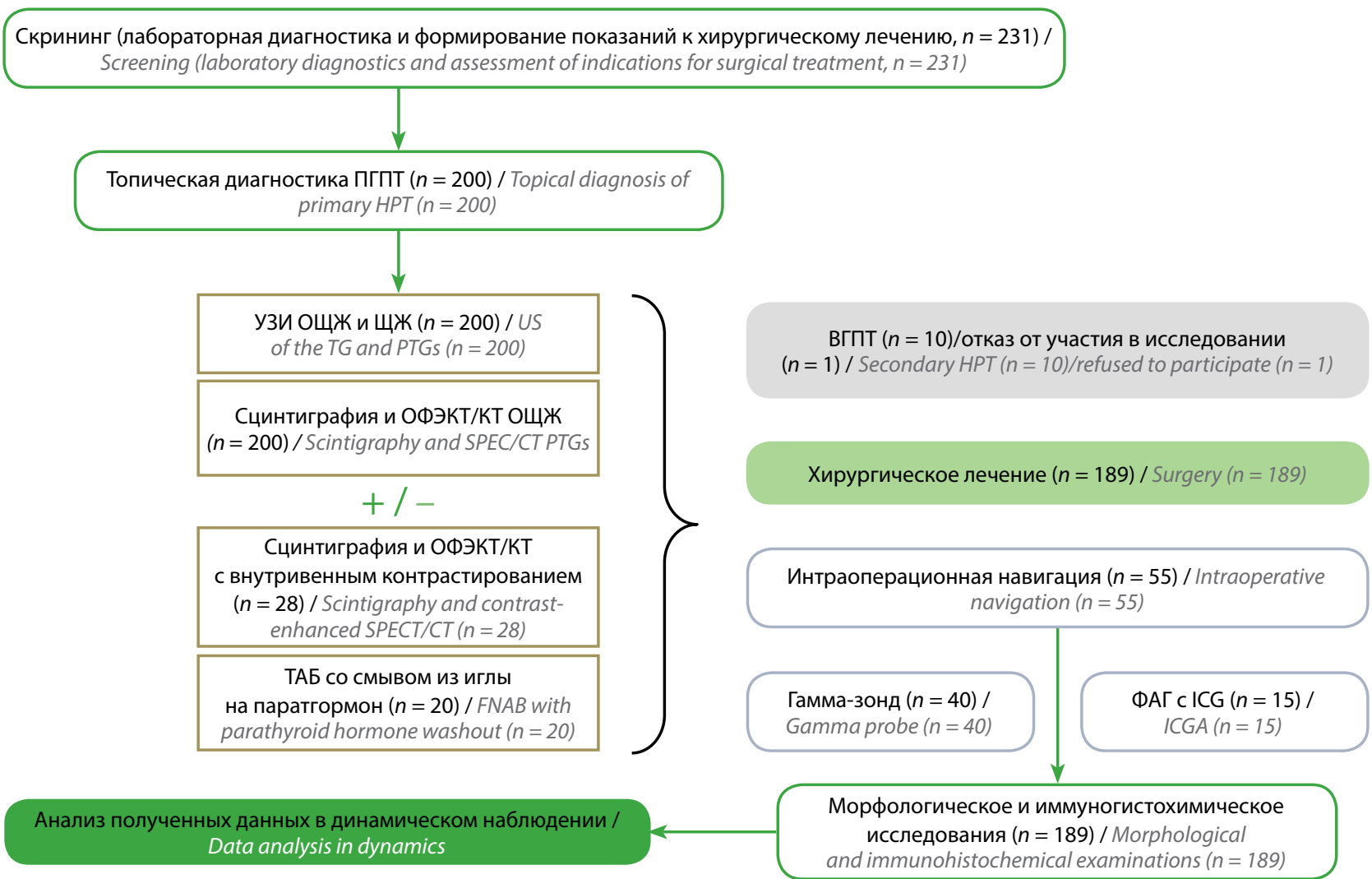

Рис. 1. Схема дизайна исследования. ПГПТ - первичный гиперпаратиреоз; ВГПТ - вторичный гиперпаратиреоз; УзИ - ультразвуковое исследование; ЩЖ - щитовидная железа; ОЩЖ - околощитовидные железы; ОФЭКТ/КТ - однофотонная эмиссионная компьютерная томография, совмещенная с компьютерной томографией; ТАБ - тонкоигольная аспирационная биопсия; ФАГ с ІСG - флюоресцентная ангиография с индоцианином зеленым

Fig. 1. Study design. HPT - hyperparathyroidism; US - ultrasound; TG - thyroid gland; PTGs - parathyroid glands; SPECT/CT - single-photon emission computed tomography/computed tomography; FNAB - fine-needle aspiration biopsy; ICGA -indocyanine green angiography

моче выявлялось только в спорных диагностических ситуациях (для исключения семейной гипокальциурической гиперкальциемии). Референсные значения лаборатории: ПТГ - 15-65 пг/мл, кальций общий 2,15-2,55 ммоль/л, кальций ионизированный 1,03-1,29 ммоль/л, фосфор - 0,74-1,52 ммоль/л.

Инструментальные исследования проводились в ФГБУ «НМИЦ эндокринологии» Минздрава России.

1. Ультразвуковое исследование ОЩЖ и ЩЖ выполняли на аппаратах Voluson E8 Expert с использованием линейного датчика (с диапазоном частот 5,0-13,0 МГц) и Toshiba (Canon) Aplio 500 с линейным датчиком (с диапазоном частот 7,0-18,0 МГц).

2. Планарную двухфазную сцинтиграфию и ОФЭКТ/КТ ОШЖ с 99m Тс-технетрилом проводили с использованием ОФЭКТ/КТ-гамма-камеры, GE Discovery NM/CT 670. При наличии показаний пациентам одномоментно выполняли внутривенное контрастирование и ОФЭКТ/КТ в раннюю или позднюю фазы исследования (планарной сцинтиграфии или ОФЭКТ/КТ).

3. Некоторым пациентам была выполнена мультиспиральная КТ ОЩЖ с внутривенным болюсным контрастированием на мультидетекторном компьютерном томографе Optima CT (General Electric, США). Толщина срезов составила 0,625 мм. Область сканирования - от основания черепа до бифуркации трахеи. Для контрастирования применяли неионное контрастное вещество йомепрол (400 мг/мл). Его вводили через катетер с помощью двухколбового автоматического инъектора со скоростью 4 мл/с 50 мл (с болюсом 40 мл 0,9\%-ного раствора $\mathrm{NaCl}$ ). Для получения артериальной и венозной фаз сканирования исследование начинали на 5-й и 20-й секундах с момента достижения порогового контрастирования аорты (с использованием технологии bolus tracking). Отсроченную фазу проводили у всех пациентов на 3-й минуте после введения контрастного препарата.

После подтверждения топической локализации образований ОЩЖ в условиях комбинированной анестезии была проведена минимально инвазивная паратиреоидэктомия. Все операции выполнены в отделе хирургии ФГБУ «НМИЦ эндокринологии» Минздрава России. В 55 случаях применяли технологии интраоперационной навигации: у 40 пациентов - гамма-зонд (Gamma 
Finder2), у 15 - флюоресцентную ангиографию с индоцианином зеленым (Novodaq, SPY 3000 ).

Патоморфологическое исследование удаленных образований ОЩЖ проводили в отделе фундаментальной патоморфологии ФГБУ «НМИЦ Эндокринологии» Минздрава России по стандартной методике. По результатам морфологического исследования были верифицированы 4 формы заболевания: аденома, гиперплазия, атипическая аденома и рак ОЩЖ.

Статистический анализ. При анализе данных для описания количественных признаков использовали медиану $(\mathrm{Me})$, нижний $(\mathrm{Q} 1)$ и верхний квартили $(\mathrm{Q} 3)$, в ряде случаев - минимальные (min) и максимальные (max) значения. Для описания качественных признаков использовали абсолютные $(n)$ и относительные (\%) частоты. Доверительные интервалы рассчитывали с помощью онлайн-калькулятора JavaStat (https://statpages.info/ctab2×2. html). Для графического отражения взаимосвязи чувствительности и специфичности методов топической диагностики были построены ROC-кривые с расчетом площади под кривой (Area Under the Curve, AUC). Для этого использовался онлайн-калькулятор MedCalc (https://www. medcalc.org/).

В ходе анализа результатов предоперационной диагностики оценивалась диагностическая информативность методов топической визуализации (независимо друг от друга и в комбинации). В зависимости от совпадения с клинико-морфологическим диагнозом или результатами динамического наблюдения все результаты подразделялись на 4 вида: истинно положительные, истинно отрицательные, ложноположительные и ложноотрицательные. Затем по специальным формулам производилось вычисление основных показателей информативности диагностического метода: ДЧ, ДС, ОТ, ПЦПР и ПЦОР. Прогностическая ценность положительного результата свидетельствует о вероятности наличия заболевания при положительном (патологическом) результате теста, а ПЦОР - о вероятности отсутствия заболевания при отрицательном (нормальном) результате теста. Референсным тестом для оценки диагностической информативности методов визуализации во всех случаях, когда было проведено хирургическое лечение, являлось гистологическое исследование удаленных образований ОЩЖ вместе с клиническим течением заболевания, подтвержденной лабораторной ремиссией ПГПТ при динамическом наблюдении за пациентом в течение не менее 6 мес. При расчете параметров информативности (ДЧ, ДС, ОТ, ПЦПР, ПЦОР) для комбинации диагностических тестов (УЗИ, ОФЭКТ/КТ \pm КТ с контрастным усилением и/или ТАБ со смывом из иглы на ПТГ) окончательный результат исследований оценивался как наличие образования ОЩЖ, если оно выявлено хотя бы одним из методов визуализации.

\section{Результаты}

Во всех случаях $(n=200)$ биохимически установлен диагноз «гиперпаратиреоз». Уровень ПТГ определен у всех больных: медиана составила 140,9 пг/мл (111,5; $218,2)$, максимальное значение - 1754 пг/мл. Количество кальция общего выявлено у подавляющего большинства пациентов $(n=198)$ : медиана составила 2,8 ммоль/л $(2,69$; $2,96)$, максимальный уровень - 3,96 ммоль/л (у 2 пациентов уровень общего кальция не определяли, но были получены данные по содержанию кальция ионизированного и суточной кальциурии). Уровень кальция ионизированного был определен у $80 \%(n=160)$ больных: медиана составила 1,37 ммоль/л $(1,32 ; 1,48)$, максимальное значение - 1,9 ммоль/л. Уровень $25(\mathrm{OH}) \mathrm{D}$ в крови проанализировано у $58 \%(n=116)$ пациентов: медиана 15,38 нг/мл $(0,0 ; 25,3)$, минимальное значение -4 нг/мл. Остальные биохимические параметры уровень фосфора $(n=64)$ и щелочной фосфатазы $(n=38)$ в крови, кальций в суточной моче $(n=25)-$ не анализировались в связи тем, что они определялись не у всех пациентов.

Возраст пациентов варьировал от 21 до 82 лет (медиана - 57 лет). Соотношение женщин и мужчин составило 11:1 (183 (91,5 \%) женщины и $17(8,5 \%)$ мужчин). В ходе обследования у 188 (94\%) пациентов диагностированы манифестные формы ПГПТ: изолированная костная - у 30 (16\%) больных, висцеральная - у 75 (40\%), смешанная - у 83 (44\%). Бессимптомная форма выявлена в 12 (6\%) случаях. Общие клинико-лабораторные характеристики пациентов представлены в табл. 1.

Таблица 1. Общие клинико-лабораторные характеристики пациентов с первичным гиперпаратиреозом

Table 1. Clinical and laboratory characteristics of patients with primary hyperparathyroidism

\begin{tabular}{|c|c|}
\hline $\begin{array}{l}\text { Показатель } \\
\text { Parameter }\end{array}$ & Me (Q1; Q3) \\
\hline $\begin{array}{l}\text { Возраст на момент лечения, лет }(n=200) \\
\text { Age at the time of treatment, years }(n=200)\end{array}$ & $57,5(51 ; 65)$ \\
\hline $\begin{array}{l}\text { Паратгормон, пг/мл }(n=200) \\
\text { Parathyroid hormone, pg/mL }(n=200)\end{array}$ & $\begin{array}{l}140,9(111,5 \\
218,2)\end{array}$ \\
\hline $\begin{array}{l}\text { Кальций общий, ммоль/л }(n=198) \\
\text { Total calcium, mmol/L }(n=198)\end{array}$ & $2,8(2,69 ; 2,96)$ \\
\hline $\begin{array}{l}\text { Кальций ионизированный, ммоль/л } \\
(n=160) \\
\text { Ionized calcium, mmol/L }(n=160)\end{array}$ & $1,37(1,32 ; 1,48)$ \\
\hline $\begin{array}{l}25(\mathrm{OH}) \mathrm{D}, \text { нг/мл }(n=116) \\
25(\mathrm{OH}) \mathrm{D}, \mathrm{ng} / \mathrm{mL}(n=116)\end{array}$ & $15,38(0,0 ; 25,3)$ \\
\hline
\end{tabular}

По данным Российского регистра по ПГПТ, средний возраст пациентов на момент постановки диагноза составил 55,6 \pm 10 лет [1]. У мужчин данное 
заболевание наблюдается всего в 9,3\% случаев. Активная форма заболевания регистрируется у $84,6 \%$ пациентов: в 48,4 \% случаев - костная форма, в $15,8 \%-$ висцеральная, в 35,8 \% - смешанная. Лабораторные показатели пациентов с ПГПТ: ПТГ - $197(134 ; 370)$ пг/мл, кальций общий $-2,86 \pm 0,34$ ммоль/л, кальций ионизированный $-1,42 \pm 0,2$ ммоль/л.

Приведенные данные сопоставимы с исследуемой нами группой пациентов, за исключением незначительно более низкого среднего уровня ПТГ и показателей распространенности различных форм ГПТ.

В нашем исследовании у 127 (63,5 \%) пациентов имелась сопутствующая структурная или функциональная патология ЩЖ: у 46 больных - узловой зоб (У3), у 45 - многоузловой зоб (МУЗ), у 5 - диффузный токсический (ДТЗ) и узловой токсический зоб (УТЗ), у $33-$ хронический аутоиммунный тиреоидит (у 21 из них УЗ сочетался с хроническим аутоиммунным тиреоидитом). В 16 (8 \%) случаях в анамнезе были операции на органах шеи, из них 6 - по поводу заболеваний ШЖ и 10 по поводу ГПТ (у 6 пациентов в связи с персистенцией ГПТ, у 4 - в связи с рецидивом заболевания).

Основные результаты исследования. Для расчета параметров диагностической информативности методов визуализации были использованы данные, полученные в ходе обследования всех 200 пациентов. В результате медицинской визуализации на предоперационном этапе у 189 больных выявлена локализация образований ОЩЖ. У 10 пациентов данных о топической локализации образований ОЩЖ получено не было. В последующем у этих больных выявлены вторичные причины повышения ПТГ. Один пациент отказался от дальнейшего участия в исследовании, несмотря на определение локализации образования ОЩЖ. Всем 189 пациентам проведено хирургическое лечение в объеме минимально инвазивной паратиреоидэктомии. В ряде случаев при сопутствующих заболеваниях ЩЖ по показаниям была выполнена паратиреоидэктомия с гемитиреоидэктомией или тотальной тиреоидэктомией.

Уровни кальция общего в крови и ПТГ нормализовались у 186 (98 \%) из 189 оперированных пациентов. У 3 больных по данным морфологического исследования не выявлено образований ОЩЖ. Результаты предоперационной диагностики (УЗИ и планарной сцинтиграфии) этих пациентов были интерпретированы неверно: определено образование ОЩЖ. При ОФЭКТ/КТ данных, свидетельствующих о наличии образований ОЩЖ, не получено.

В 48 (24 \%) случаях, помимо УЗИ, планарной сцинтиграфии и ОФЭКТ/КТ, потребовалось дополнительное проведение КТ или ОФЭКТ/КТ с внутривенным контрастированием $(n=28)$ и пункционной биопсии кандидатного образования со смывом из иглы на ПТГ $(n=20)$ для определения объекта, подлежащего прецизионному хирургическому лечению. Длительность наблюдения после операции составила не менее 6 мес. За этот период у пациентов, получивших хирургическое лечение, не выявлено ни одного рецидива или персистенции заболевания. Под динамическим наблюдением находились 10 из 200 пациентов с вторичными причинами повышения ПТГ, которым не было выполнено хирургическое лечение. Во всех случаях наблюдалась положительная динамика на фоне проводимой консервативной терапии. Один пациент отказался от дальнейшего участия в исследовании, и не был прооперирован. Результаты, полученные в ходе инструментальной диагностики этих 11 пациентов, также вошли в финальный анализ данных диагностической информативности методов предоперационной визуализации (табл. 2).

Важно отметить высокие показатели ДС и ПЦОР ОФЭКТ/КТ по сравнению с другими методами визуализации. Это является основным критерием выбора дальнейшей лечебной тактики в отношении пациентов с ПГПТ. За счет применения дополнительных методов предоперационной визуализации удается повысить показатели ДЧ и ОТ, что позволяет рекомендовать комбинацию методов в большинстве случаев при планировании минимально инвазивной паратиреоидэктомии.

Дополнительно для иллюстрации чувствительности и специфичности методов предоперационной диагностики изолированно друг от друга и в комбинации были построены ROC-кривые (рис. 2-5). На практике для диагностического поиска патологически измененных ОЩЖ лучше использовать ОФЭКТ/КТ и УЗИ, дополненные КТ с контрастным усилением и/или ТАБ со смывом из иглы на ПТГ. Комбинация этих методов имеет лучшие показатели ДЧ, ОТ и ПЦОР.

Дополнительные результаты исследования. Диагноз подтвержден гистологическим исследованием у 186 из 189 прооперированных пациентов. В 173 (93\%) случаях выявлена аденома, в $5(2,7 \%)$ - гиперплазия ОЩЖ; в $5(2,7 \%)$ - рак ОЩЖ; в $3(1,6 \%)$ - атипическая аденома ОЩЖ.

По данным предоперационной визуализации и морфологического исследования образования ОЩЖ в $52(28 \%)$ случаях локализовались в верхней ОЩЖ, в $99(51 \%)$ - в нижней ОЩЖ. У 26 (14\%) пациентов ОЩЖ были расположены атипично (в трахеопищеводной борозде - 12; в рогах тимуса -8 ; интратиреоидно -3 ; в средостении -2 ; в поднижнечелюстной области - 1). Множественное поражение ОЩЖ наблюдалось у 15 (7 \%) больных.

У $3(<2 \%)$ из 189 пациентов в результате патоморфологического исследования не было обнаружено образований ОЩЖ. Из них у 2 больных выявлены фолликулярные аденомы ЩЖ (в 1 случае имелась также папиллярная микрокарцинома ЩЖ (рT1a), в 1 случае ткань ЩЖ была нормального гистологического строения, ОЩЖ не изменены), которые при предоперационной топической диагностике ошибочно приняты 
Таблица 2. Диагностическая информативность методов предоперационной диагностики

Table 2. Performance characteristics of different methods of preoperative diagnostics

\begin{tabular}{|c|c|c|c|c|c|}
\hline $\begin{array}{l}\text { Метод диагностики } \\
\text { Diagnostic method }\end{array}$ & $\begin{array}{c}\text { Диагностическая } \\
\text { чувствительность } \\
\text { (95\% ДИ) } \\
\text { Diagnostic sensitivity } \\
(95 \% \text { CI) }\end{array}$ & $\begin{array}{c}\text { Диагностическая } \\
\text { специфичность } \\
\text { (95 \% ДИ) } \\
\text { Diagnostic specificity } \\
\text { (95\% CI) }\end{array}$ & $\begin{array}{c}\text { Общая } \\
\text { точность } \\
\text { (95\% ДИ) } \\
\text { Overall accuracy } \\
(95 \% \text { CI) }\end{array}$ & $\begin{array}{c}\text { Прогностическая } \\
\text { ценность } \\
\text { положительного } \\
\text { результата } \\
\text { (95\% ДИ) } \\
\text { Positive predictive } \\
\text { value (95\% CI) }\end{array}$ & $\begin{array}{c}\text { Прогностическая } \\
\text { ценность } \\
\text { отрицательного } \\
\text { результата } \\
\text { (95\% ДИ) } \\
\text { Negative predictive } \\
\text { value }(95 \% \text { CI) }\end{array}$ \\
\hline $\begin{array}{l}\text { Планарная сцинтиграфия } \\
\text { Planar scintigraphy }\end{array}$ & $69(63-76)$ & $57(31-100)$ & $69(62-75)$ & $96(92-100)$ & $12(4-20)$ \\
\hline $\begin{array}{l}\text { ОФЭКТ } / \text { КТ } \\
\text { SРЕСТ } / \text { CT }\end{array}$ & $91(87-95)$ & $92(78-100)$ & $91(87-95)$ & $99(98-100)$ & $41(23-100)$ \\
\hline $\begin{array}{l}\text { УЗИ } \\
\text { US }\end{array}$ & $84(79-89)$ & $38(12-65)$ & $81(76-86)$ & 95 (92-98) & $14(3-26)$ \\
\hline $\begin{array}{l}\text { УЗИ + ОФЭКТ/КТ (+ КТ } \\
\text { с контрастным усилением } \\
\text { и/или ТАБ со смывом } \\
\text { на ПТГ) } \\
\text { US + SPECТ /CT (contrast- } \\
\text { enhanced CT and/or FNAB } \\
\text { with PTH washout) }\end{array}$ & $100(98-100)$ & $77(54-100)$ & $99(97-100)$ & $98(97-100)$ & $100(98-100)$ \\
\hline
\end{tabular}

Примечание. ОФЭКТ/КТ - однофотонная эмиссионная компьютерная томография, совмещенная с компьютерной томографией; УЗИ - ультразвуковое исследование; ТАБ - тонкоигольная аспирационная биопсия; ПТГ - паратгормон; ДИ - доверительный интервал.

Note. SPECT/CT - single-photon emission computed tomography/computed tomography; US - ultrasound; FNAB - fine-needle aspiration biopsy; PTH - parathyroid hormone; $C I$ - confidence interval.

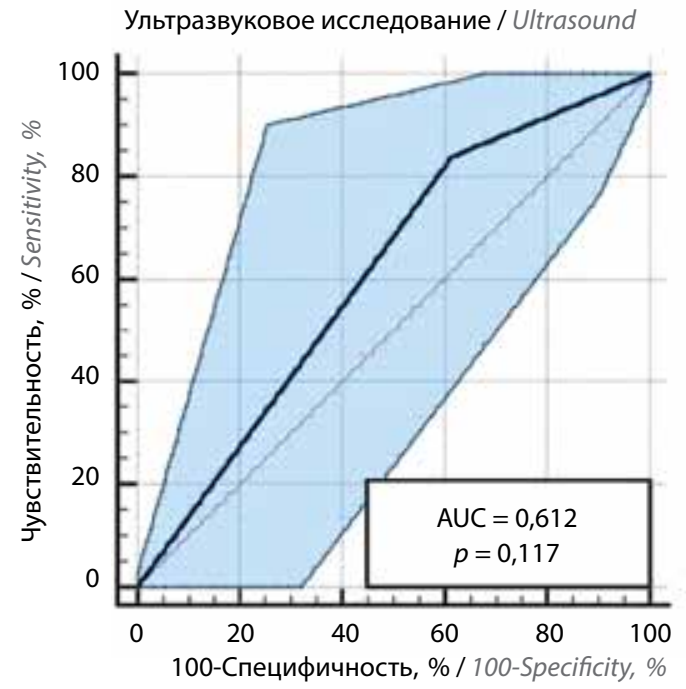

Рис. 2. Чувствительность и специфичность ультразвукового исследования, выполняемого для предоперационной диагностики первичного гиперпаратиреоза

Fig. 2. Sensitivity and specificity of ultrasound in preoperative diagnosis of primary hyperparathyroidism

за образования ОЩЖ. Уровень кальция после операции нормализовался, но сохранялось повышение уровня ПТГ. Все 3 пациента получали консервативную терапию, которая имела положительный эффект, в связи

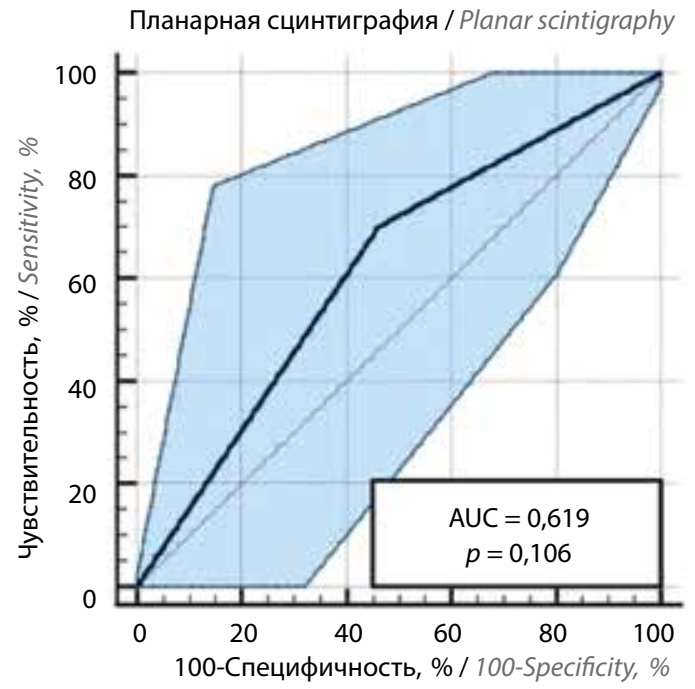

Рис. 3. Чувствительность и специфичность планарной сцинтиграфии, выполняемой для предоперационной диагностики первичного гиперпаратиреоза

Fig. 3. Sensitivity and specificity of planar scintigraphy in preoperative diagnosis of primary hyperparathyroidism

с чем предположен вторичный генез ГПТ. Больные находятся под динамическим наблюдением.

Множественные образования ОЩЖ (от 2 до 4 патологически измененных желез) выявлены в 15 случаях. 
Однофотонная эмиссионная компьютерная томография, совмещенная с компьютерной томографией / Single-photon emission computed tomography/computed tomography

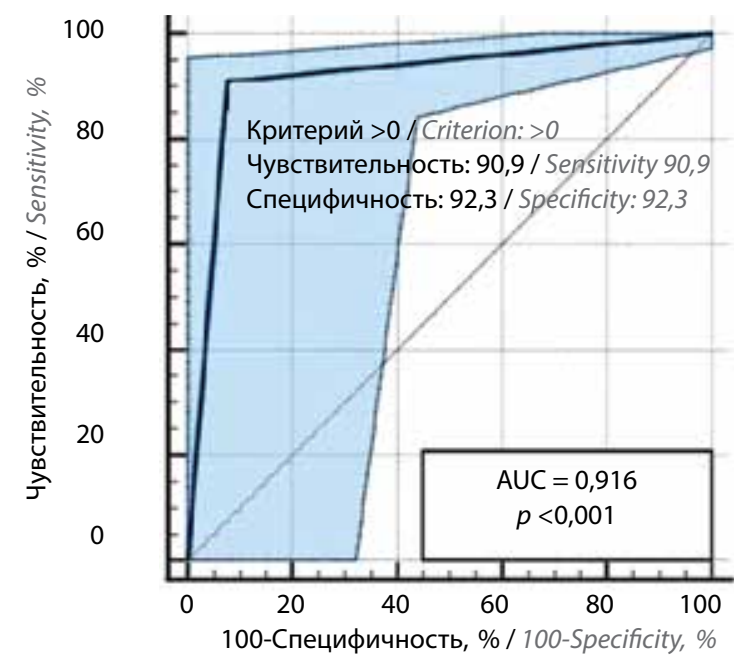

Рис. 4. Чувствительность и специфичность однофотонной эмиссионной компьютерной томографии, совмещенной с компьютерной томографией, выполняемой для предоперационной диагностики первичного гиперпаратиреоза

Fig. 4. Sensitivity and specificity of single-photon emission computed tomography/computed tomography in preoperative diagnosis of primary hyperparathyroidism

Размеры опухолей варьировали от 0,5 до $8 \mathrm{~cm}$. В ходе нашего исследования при размере образований ОЩЖ от 1 до 2 см в 1 случае выявлен рак ОЩЖ (однако это была не первичная опухоль, а местный рецидив после ранее проведенного хирургического лечения размерами 2 см в диаметре), при размере образований от 2 до $3 \mathrm{~cm}$ - также 1 случай рака ОЩЖ (первичная опухоль, 3 см в диаметре), при размере образований $>3$ см в диаметре - 3 случая рака ОЩЖ и 3 атипические аденомы ОЩЖ (опухоли от 4,5 до 6,0 см в диаметре), что подтверждает опубликованные ранее данные о более высокой вероятности рака ОЩЖ при размере образования ОЩЖ $\geq 3$ см. Образования ОЩЖ преимущественно состоят из главных клеток, а также представлены смешанно-клеточными вариантами. В единичных случаях были выявлены аденомы с преобладанием оксифильных клеток.

Интраоперационная навигация. В ходе эксперимента определена оптимальная активность ${ }^{99 \mathrm{~m}}$ Тс-МИБИ для проведения операций с гамма-навигацией на тестовой выборке из 10 пациентов с целью выявления закономерности очагового и фонового накопления радиофармацевтического препарата (РФП). Навигационная активность ${ }^{99 \mathrm{~m}} \mathrm{Tc}-$ МИБИ (от 55 до 450 МБк) вводилась за 30-180 мин до операции. Поиск образования ОЩЖ производили визуально, учитывая данные предоперационной топической диагностики (УЗИ, КТ, сцинтиграфии, ОФЭКТ/КТ). Гамма-зонд использовали в качестве подтверждения удаления функциониру-
Комбинация методов / Combination of methods

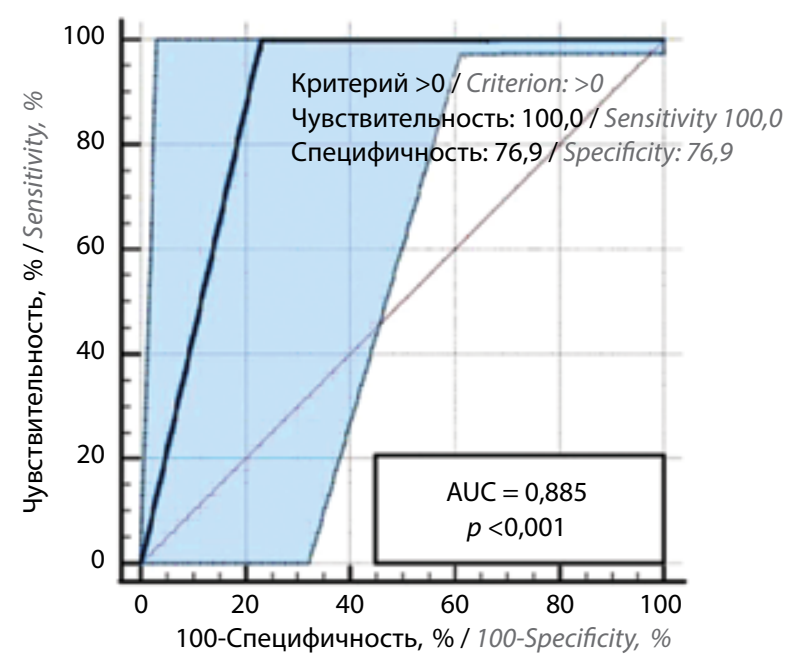

Рис. 5. Чувствительность и специфичность комбинации ультразвукового исследования и однофотонной эмиссионной компьютерной томографии, совмещенной с компьютерной томографией, дополненных компьютерной томографией с контрастным усилением и/или тонкоигольной аспирационной биопсии со смывом из иглы на паратгормон

Fig. 5. Sensitivity and specificity of combination of ultrasound plus singlephoton emission computed tomography/computed tomography plus contrastenhanced computed tomography and/or fine-needle aspiration biopsy with parathyroid hormone washout

ющей ткани ОЩЖ. После ее удаления в течение 10 мин проводили исследование in vitro удаленной ткани в калибраторе доз. При этом регистрировалась остаточная активность радиофармацевтических препаратов в ткани удаленной ОЩЖ, что подтверждало полученные с помощью прибора данные. В ходе исследования измеряли показатели фонового излучения до операции в предполагаемой зоне, фон от сердца (счет 140-190), мочевого пузыря (счет 100-150), нижних конечностей (счет 10-15), а также в операционном поле (ЩЖ, ОЩЖ, аденома ОЩЖ, мышцы, жировая ткань). После анализа тестовых данных 10 пациентов наблюдалось уменьшение погрешности (девиации) измерения счета при увеличении введенной активности (150-300 МБк) и времени до измерения погрешности счета (излучения) во время операции. Таким образом, была определена оптимальная активность $99 \mathrm{~m}$ Тс-МИБИ. Рекомендуется вводить 300 МБк ${ }^{99 \mathrm{~m}}$ Тс-МИБИ за 60-90 мин до операции (перед премедикацией). По данной методике выполнено 30 операций с применением одноканального гамма-зонда. В $100 \%$ случаев было достигнуто снижение уровня излучения после удаления функционально активного образования ОЩЖ на $20 \%$ и более, что свидетельствует о радикальности хирургического лечения. Важно отметить, что применение гамма-зонда позволяет идентифицировать образования ОЩЖ интраоперационно, еще до их удаления, но в большей степени оно информативно при верификации уже удаленных кандидатных образований. 
Специальный отбор пациентов отсутствовал. В исследование в случайном порядке были включены 30 пациентов с положительными результатами планарной сцинтиграфии и ОФЭКТ/КТ с ${ }^{99 \mathrm{~m}} \mathrm{Tc}-$ МИБИ. В качестве дополнительного контроля радикальности хирургического лечения интраоперационно определяли уровень ПТГ $\left(0^{\prime}, 15^{\prime}\right.$ после удаления образования ОЩЖ) и выполняли плановое гистологическое исследование удаленного материала. В 6 случаях из 30 ход операции был изменен (стала проводиться односторонняя ревизия мест типичного расположения ОЩЖ) в связи с отсутствием снижения уровня излучения после удаления одного кандидатного образования по данным, получаемым с гамма-зонда.
Анализ результатов 15 операций, выполненных по поводу ПГПТ с применением флюоресцентной ангиографии с индоцианином зеленым, показал, что данная методика, применяемая с целью поиска измененных ОЩЖ, имеет ограничения в связи с активным кровоснабжением близлежащих структур на шее (это затрудняет дифференциальную диагностику образований). При ПГПТ метод флюоресцентной ангиографии с индоцианином зеленым может быть наиболее эффективен в случае хирургического лечения после ранее проведенной тиреоидэктомии/гемитиреоидэктомии, если образование ОЩЖ локализуется на стороне удаленной доли ЩЖ. Альтернативная область применения вышеуказанной методики - прогнозирование риска

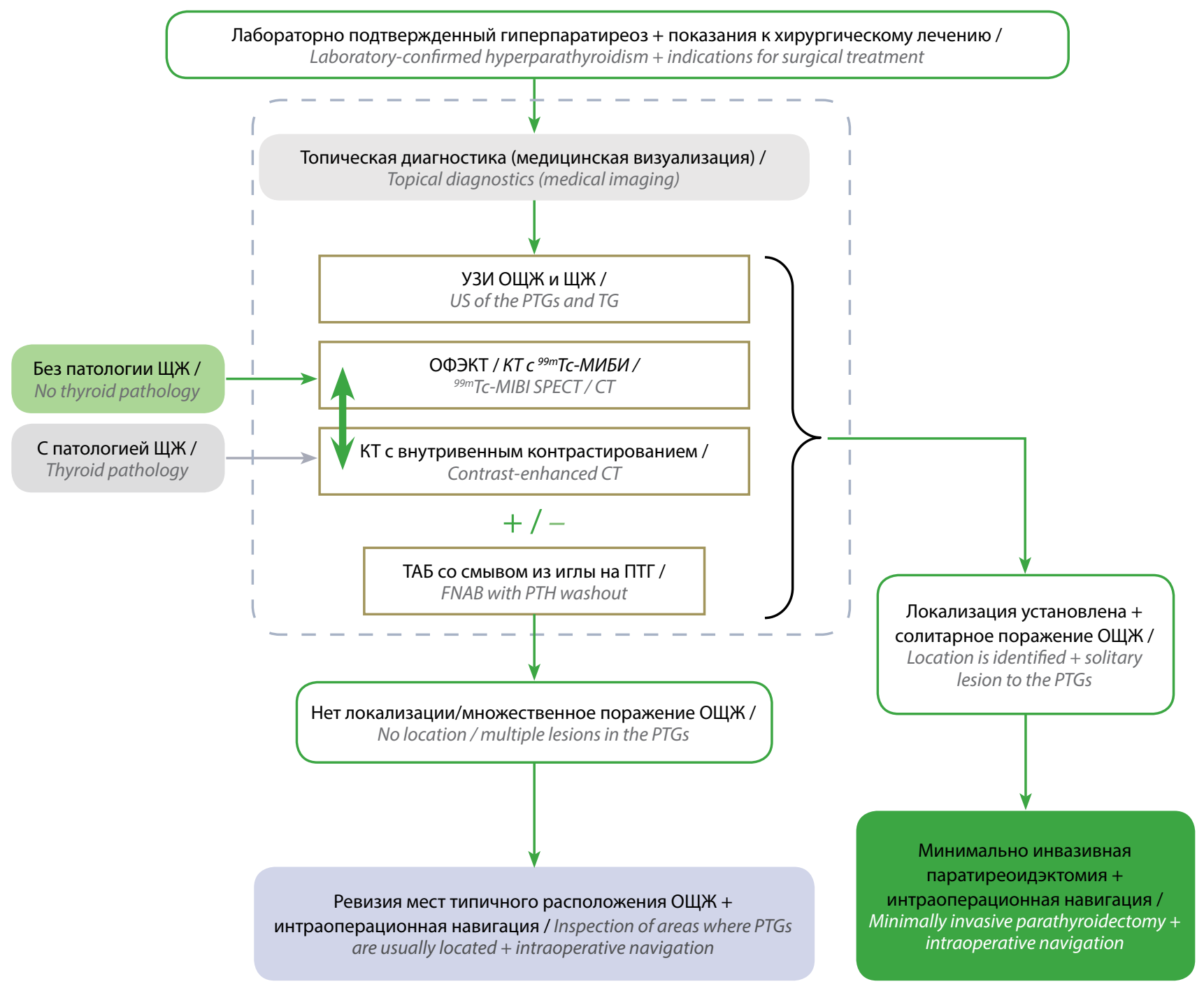

Рис. 6. Алгоритм предоперационной топической диагностики образований околощитовидных желез (ОЩЖ) при первичном гиперпаратиреозе. ЩЖ - щитовидная железа; КТ - компьютерная томография; ТАБ - тонкоигольная аспирационная биопсия; ОФЭКТ/КТ - однофотонная эмиссионная компьютерная томография, совмещенная с компьютерной томографией; ПТГ- паратгормон; УзИ-ультразвуковое исследование Fig. 6. Algorithm of preoperative topical diagnosis of parathyroid tumors in patients with primary hyperparathyroidism. TG - thyroid gland; CT - computed tomography; SPECT/CT - single-photon emission computed tomography/computed tomography; PTH - parathyroid hormone; US - ultrasound; PTGs parathyroid glands 
развития ГПТ после операций на ЩЖ или на ЩЖ и ОЩЖ), особенно при наличии в анамнезе хирургических вмешательств на органах шеи в связи с рецидивом или персистенцией заболевания.

\section{Обсуждение}

Выбор какого-то одного оптимального диагностического метода крайне затруднителен, поскольку ни один из них не обладает $100 \%$-ной диагностической точностью. К тому же каждое исследование имеет ограничения, а его результативность в значительной степени зависит от квалификации специалиста $[4,5,7]$. Полученные нами данные позволили выделить комбинацию наиболее информативных диагностических методов, использующихся на предоперационном этапе, а также показали возможность применения современных технологий интраоперационной навигации в эндокринной хирургии. Локализация измененных ОЩЖ была правильно распознана у 186 (98 \%) из 189 пациентов с ПГПТ и показаниями к хирургическому лечению с помощью УЗИ ОЩЖ в сочетании с ОФЭКТ/КТ. Это позволяет рекомендовать данную наиболее информативную комбинацию комплементарных друг другу методов в качестве стандарта диагностики у больных ПГПТ. Стоит также отметить, что в $24 \%$ случаев $(n=48)$ предлагаемую комбинацию диагностических исследований потребовалось дополнить либо ОФЭКТ/КТ или КТ с внутривенным контрастированием (в 28 (14 \%) случаях), либо пункционной биопсией образований со смывом из иглы на ПТГ (в 20 (10 \%) случаев). Во всех случаях у пациентов была диагностирована сопутствуюшая структурная или функциональная патология ЩЖ.

Полученные нами данные указывают на значительное влияние функциональной (хронический аутоиммунный тиреоидит, УТЗ или ДТЗ) и узловой (УЗ и МУЗ, включая рак щитовидной железы) патологии ЩЖ на интерпретацию результатов предоперационной диагностики, что соответствует данным ряда зарубежных авторов [13, 14].

Следует отметить, что изолированная информативность планарной сцинтиграфии и КТ без контрастного усиления является крайне низкой, поэтому мы не рекомендуем выполнять эти исследования для предоперационной диагностики у пациентов с ПГПТ. Применение ТАБ со смывом из иглы на ПТГ под контролем УЗИ рекомендуется в качестве дополнительного теста при необходимости верификации кандидатных образований ОЩЖ и не может использоваться в качестве самостоятельного метода предоперационной диагностики.

В эпоху минимально инвазивной хирургии технологии интраоперационной навигации имеют широкую область применения в эндокринной хирургии. Интраоперационный гамма-зонд возможно использовать в ходе минимально инвазивной паратиреоидэктомии при положительном результате планарной сцинтиграфии

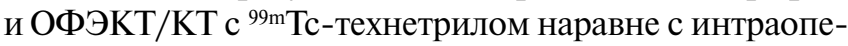
рационным определением уровня ПТГ. Это позволит снизить частоту нерадикальных хирургических вмешательств, существенно не увеличивая время операции. Флюоресцентная ангиография с индоцианином зеленым позволяет визуализировать кровоснабжение ОЩЖ в режиме реального времени и может применяться для оценки их жизнеспособности после операций на ЩЖ.

Резюме основного результата исследования. Результаты нашего исследования позволили разработать алгоритм предоперационной топической диагностики образований ОЩЖ у пациентов с ПГПТ (рис. 6).

Ограничения исследования. Основными недостатками настоящего исследования являются ограниченная в связи со сроками выполнения плана научно-исследовательской работы клиническая выборка пациентов, недоступность позитронно-эмиссионной томографии, совмещенной с KT, c ${ }^{18} \mathrm{~F}$-холином (или ${ }^{11} \mathrm{C}$-метионином), обусловленная крайне низкой распространенностью данного исследования в России, а также неиспользование в качестве рутинного теста определения уровней кальциурии и альбумин-скорректированного кальция крови для всех пациентов с подозрением на ПГПТ в нашей выборке. Также в данной работе не анализировались такие важные при оценке медицинских изображений параметры, как сходимость и воспроизводимость инструментальных методов, что, несомненно, имеет существенное значение и требует отдельных исследований.

\section{Заключение}

Для топической диагностики образований ОЩЖ при ПГПТ мы рекомендуем использовать следующий алгоритм действий. После лабораторного подтверждения диагноза и при наличии показаний к хирургическому лечению на 1-м этапе всем пациентам нужно выполнить УЗИ ЩЖ и ОЩЖ. На 2-м этапе (при отсутствии выраженных изменений в ШЖ) необходимо выполнить ОФЭКТ /КТ с ${ }^{99 \mathrm{~m}} \mathrm{Tc}-$ МИБИ и при совпадении результатов 2 диагностических тестов приступить к минимально инвазивной паратиреоидэктомии с интраоперационной навигацией. При сомнительных результатах следует провести КТ-исследование с внутривенным контрастированием или ТАБ со смывом из иглы на ПТГ. При наличии сопутствующей функциональной или структурной патологии ЩЖ на 2-м этапе КТ с внутривенным контрастированием при необходимости можно дополнить ОФЭКТ/КТ с ${ }^{99 \mathrm{~m}} \mathrm{Tc}-$ МИБИ и/или ТАБ со смывом из иглы на ПТГ.

Радионавигационная хирургия с применением одноканального гамма-зонда ( ${ }^{99 \mathrm{~m}} \mathrm{Tc}-$ МИБИ) рекомендована для использования в ходе минимально инвазив- 
ной паратиреоидэктомии при условии положительного результата планарной сцинтиграфии и/или ОФЭКТ/КТ c ${ }^{99 \mathrm{~m}}$ Тс-МИБИ на предоперационном этапе в качестве метода, позволяющего быстро верифицировать удаля- емые образования ОЩЖ. Флюоресцентную ангиографию с индоцианином зеленым можно использовать для оценки кровоснабжения ОЩЖ при операциях на ЩЖ.

\section{Л И Т E P A T У}

1. Мокрышева Н.Г., Мирная С.С., Добрева Е.А. и др. Первичный гиперпаратиреоз в России по данным регистра. Проблемы эндокринологии 2019;65(50):300-10. [Mokrysheva N.G., Mirnaya S.S., Dobreva E.A. et al. Primary hyperparathyroidism in Russia according to the register. Problemy endokrinologii $=$ Problems of Endocrinology 2019;65(50):300-10. (In Russ.)]. DOI: $10.14341 /$ probl10126.

2. Yeh M.W., Ituarte P.H.G., Zhou H.C. et al. Incidence and prevalence of primary hyperparathyroidism in a racially mixed population. J Clin Endocrin Metab 2013;98(3):1122-9. DOI: $10.1210 /$ jc. 2012-4022.

3. Sudhaker D. Epidemiology of parathyroid disorders. Best Pract Res Clin Endocrinol Metab 2018;32(6):773-80. DOI: $10.1016 /$ j.beem.2018.12.003.

4. Слашук К.Ю., Дегтярев М.В., Румянцев П.О. и др. Методы визуализации околощитовидных желез при первичном гиперпаратиреозе. Обзор литературы. Эндокринная хирургия 2019;13(4):153-74. [Slashchuk K.Yu., Degtyarev M.V., Rumyantsev P.O. et al. Methods of visualization of the parathyroid glands in primary hyperparathyroidism. Literature review. Endocrine Surgery = Endokrinnaya hirurgiya
2019;13(4):153-74. (In Russ.)]. DOI: $10.14341 / \operatorname{serg} 12241$.

5. Cheung K., Wang T.S., Farrokhyar F. et al. A meta-analysis of preoperative localization techniques for patients with primary hyperparathyroidism. Ann Surg Oncol 2012;19(2):577-83.

6. Kluijfhout W.P., Pasternak J.D., Beninato T. et al. Diagnostic performance of computed tomography for parathyroid adenoma localization; a systematic review and meta-analysis. Eur J Radiol 2017;88:117-28. DOI: 10.1016/j.ejrad.2017.01.004.

7. Baj J., Sitarz R., Łokaj M. et al. Preoperative and intraoperative methods of parathyroid gland localization and the diagnosis of parathyroid adenomas. Molecules 2020;25(7):1724. DOI: $10.3390 /$ molecules25071724.

8. Lombardi C.P., Raffaelli M., Traini E. et al. Video-assisted minimally invasive parathyroidectomy: benefits and long-term results. World J Surg 2009;33(11):2266. DOI: 10.1007/s00268-009-9931-7.

9. Suliburk J.W., Sywak M.S., Sidhu S.B., Delbridge L.W. 1000 minimally invasive parathyroidectomies without intra-operative parathyroid hormone measurement: lessons learned. ANZ J Surg 2011;81(5):362-5. DOI: 10.1111/j.1445-2197.2010.05488.x.
10. Udelsman R., Lin Z., Donovan P. et al. The superiority of minimally invasive parathyroidectomy based on 1650 consecutive patients with primary hyperparathyroidism. Ann Surg 2011;253(3):585-91. DOI: $10.1097 /$ SLA.0b013e318208fed9.

11. Venkat R., Kouniavsky G., Tufano R.P. et al. Long-term outcome in patients with primary hyperparathyroidism who underwent minimally invasive parathyroidectomy. World J Surg 2012;36(1):55-60. DOI: $10.1007 / \mathrm{s} 00268-011-1344-8$.

12. Wilhelm S.M., Wang T.S., Ruan D.T. et al. The American Association of Endocrine Surgeons Guidelines for Definitive Management of Primary Hyperparathyroidism. JAMA Surg 2016;151(10):959-68. DOI: 10.1001/jamasurg.2016.2310.

13. Lorberboym M., Ezri T., Schachter P.P. Preoperative technetium $\mathrm{Tc}^{99 \mathrm{~m}}$ sestamibi SPECT imaging in the management of primary hyperparathyroidism in patients with concomitant multinodular goiter. Arch Surg 2005;140(7):656-60. DOI: 10.1001/archsurg.140.7.656.

14. Shafiei B., Hoseinzadeh S., Fotouhi F. et al. Preoperative ${ }^{99 \mathrm{~m}} \mathrm{Tc}$-sestamibi scintigraphy in patients with primary hyperparathyroidism and concomitant nodular goiter: comparison of SPECT-CT, SPECT, and planar imaging. Nucl Med Commun 2012;33(10):1070-6.

DOI: 10.1097/MNM.0b013e32835710b6.

Благодарности. Авторы выражают сердечную благодарность сотрудникам ФГБУ «Научный медицинский исследовательский центр эндокринологии» Минздрава России, принимающим непосредственное участие в проведении исследования: радиологам и медицинским физикам (С.С. Серженко, А.А. Бубнову, А.А. Трухину, Я.И. Сироте); хирургам (Н.С. Кузнецову, В.Э. Ванушко, К.В. Ланщакову, А.Н. Гадзыре, Д.А. Деркачу); специалистам по ультразвуковой диагностике (С.М. Захарову, Т.В. Солдатову, А.М. Артемову); эндокринологам (Ю.А. Крупиновой, А.М. Горбачевой, Е.О. Мамедовой, Л.К. Дзерановой и др.); патоморфологам (Л.С. Селивановой, А.Ю. Абросимову); сотрудникам лаборатории (Л.В. Никанкиной и др.); директору Института клинической эндокринологии Е.А. Трошиной.

Acknowledgments. The authors express their heartfelt gratitude to the staff of the Scientific Medical Research Center of Endocrinology, Ministry of Health of Russia, who are directly involved in the study: radiologists and medical physicists (S.S. Serzhenko, A.A. Bubnov, A.A. Trukhin, Ya.I. Sirota); surgeons (N.S. Kuznetsov, V.E. Vanushko, K.V. Lanshchakov, A.N. Gadzyra, D.A. Derkach); specialists in ultrasound diagnostics (S.M. Zakharov, T.V. Soldatov, A.M. Artemov); endocrinologists (Yu.A. Krupinova, A.M. Gorbacheva, E.O. Mammadova, L.K. Dzeranova et al.); pathologists (L.S. Selivanova, A.Y. Abrosimov); laboratory staff (L.V. Nikankina et al.); Director of the Institute of Clinical Endocrinology E.A. Troshina.

\section{Вклад авторов}

К.Ю. Слашук, М.В. Дегтярев: концепция и дизайн исследования, написание текста статьи, оформление иллюстративного материала, сбор и обработка материала;

П.О. Румянцев: концепция и дизайн исследования, написание текста статьи;

А.К. Еремкина, Н.В. Тарбаева, И.В. Ким: сбор материала, написание текста статьи;

Д.Г. Бельцевич: дизайн исследования, сбор материала, написание текста статьи;

Г.А. Мельниченко, Н.Г. Мокрышева: дизайн исследования, редактирование статьи. 
Authors' contributions

K.Yu. Slashchuk, M.V. Degtyarev: developing the reserch and concept desing, article writing, design of the illustrative material, obtaining data for analysis, analysis of the obtained data;

P.O. Rumyantsev: developing the reserch and concept desing, article writing;

A.K. Eremkina, N.V. Tarbaeva, I.V. Kim: obtaining data for analysis, article writing;

D.G. Beltsevich: developing the reserch desing, obtaining data for analysis, article writing;

G.A. Melnicthhenko, N.G. Mokrysheva: developing the reserch desing, text editing.

ORCID авторов / ORCID of authors

К.Ю. Слащук / K.Yu. Slashchuk: https://orcid.org/0000-0002-3220-2438

M.В. Дегтярев / M.V. Degtyarev: https://orcid.org/0000-0001-5652-2607

П.О. Румянцев / Р.О. Rumyantsev: https://orcid.org/0000-0002-7721-634X

А.К. Еремкина / А.K. Eremkina: https://orcid.org/0000-0001-6667-062X

H.B. Тарбаева / N.V. Tarbaeva: https://orcid.org/0000-0001-7965-9454

И.В. Ким / I.V. Kim: https://orcid.org/0000-0001-7552-259X

Д.Г. Бельцевич / D.G. Beltsevich: https://orcid.org/0000-0001-7098-4584

Г.А. Мельниченко / G.A. Melnicthhenko: https://orcid.org/0000-0002-5634-7877

Н.Г. Мокрышева / N.G. Mokrysheva: https://orcid.org/0000-0002-9717-9742

Конфликт интересов. Авторы заявляют об отсутствии конфликта интересов.

Conflict of interest. The authors declare no conflict of interest.

Финансирование. Работа проведена в рамках клинической апробации Минздрава России «Оказание медицинской помощи пациентам с первичным и вторичным гиперпаратиреозом на основе гибридных технологий радионуклидной визуализации и гамма-навигационной хирургии», выполняемой на базе ФГБУ «Научного медицинского исследовательского центра эндокринологии» Минздрава России в период с 2018 по 2020 г. (№ 2018-4-1).

Financing. This study was performed as a part of the clinical program "Providing medical care to patients with primary and secondary hyperparathyroidism using hybrid technologies of radionuclide imaging and gamma-navigation surgery" at the Research Medical Center of Endocrinology, Ministry of Health of Russia between 2018 and 2020 (No. 2018-4-1).

Соблюдение прав пациента и правил биоэтики

Работа выполнена при одобрении локального этического комитета (протокол № 22 от 13.12.2017) и в рамках клинической апробации Минздрава России по протоколу «Оказание медицинской помощи пациентам с первичным и вторичным гиперпаратиреозом на основе гибридных технологий радионуклидной визуализации и гамма-навигационной хирургии» (№ 2018-4-1). Все пациенты подписали добровольное информированное согласие на участие в исследовании.

Compliance with patient right and principles of bioethics

The study protocol was by the local ethics committee (protocol No. 22 dated 13.12.2017) as a part of the clinical program according to the protocol "Providing medical care to patients with primary and secondary hyperparathyroidism using hybrid technologies of radionuclide imaging and gammanavigation surgery" (No. 2018-4-1). All patients signed an informed consent to participate in this study.

Статья поступила: 04.10.2021. Принята к публикации: 29.10.2021.

Article submitted: 04.10.2021. Accepted for publication: 29.10.2021. 